\title{
DISEÑO Y VALIDACIÓN DE UNA ESCALA PARA MEDIR LA PERCEPCIÓN SOBRE EL TRABAJO EN EL PRIMER NIVEL DE ATENCIÓN EN ESTUDIANTES DE MEDICINA DE LATINOAMÉRICA
}

\author{
Percy Mayta-Tristán ${ }^{1,2, a}$, Edward Mezones-Holguín ${ }^{1,3, b}$, Reneé Pereyra-Elías ${ }^{1,4, c}$, \\ Juan J. Montenegro-Idrogo ${ }^{2, c}$, Christian R. Mejia ${ }^{1,5, d}$, Andrés Dulanto-Pizzorni', \\ Sergio R. Muñoz ${ }^{6, e}$, Red LIRHUS*
}

RESUMEN

Objetivos. Diseñar y validar una escala para evaluar la percepción sobre el primer nivel de atención (PNA) en estudiantes de medicina latinoamericanos. Materiales y métodos. Se realizó un estudio multicéntrico, observacional, en dos fases: i) Se diseñó un cuestionario autoaplicado referente a la percepción sobre la labor del médico en el PNA, y ii) Se aplicó el cuestionario en estudiantes de medicina de 18 universidades de ocho países hispanohablantes latinoamericanos. Se desarrolló un análisis factorial exploratorio (AFE) por medio de un análisis de componentes principales con rotación ortogonal varimax. Se evaluó la adecuación y el ajuste de la muestra. La extracción de factores fue hecha en base a los criterios de Kaiser, la pendiente de Catell y la varianza explicada $(>5 \%)$. La consistencia interna fue medida con el alfa de Cronbach. Resultados. Se incluyeron 423 estudiantes, el $53,4 \%$ pertenecían a universidades peruanas. Tras el AFE el cuestionario quedo constituido por 11 ítems, los cuales se distribuyen en tres dominios que, en su conjunto, explican el 55,47\% de la varianza total. i) Percepciones sobre el médico que trabaja en PNA, ii) Percepciones sobre el trabajo asistencial en PNA y iii) Percepciones sobre las consecuencias económicas de trabajar en PNA. Conclusiones. La escala presenta tres dominios y constituye un instrumento que puede ser usado para medir las percepciones sobre el trabajo médico en el primer nivel de atención en estudiantes de medicina latinoamericanos de habla hispana.

Palabras clave: Atención primaria de salud; Recursos humanos en salud; Educación médica; Análisis factorial; Latinoamérica (Fuente: DeCS BIREME)

\section{DESIGN AND VALIDATION OF A SCALE TO ASSESS LATIN AMERICAN MEDICAL STUDENTS' PERCEPTION ON THE LABOUR OF THE FIRST LEVEL OF HEALTH CARE}

\begin{abstract}
Objectives. To design and validate a scale to assess Latin American medical students' perception on first level of health care (FLHC). Materials and methods. An observational, analytic and multicentre study was carried out in two phases: i) A self-administered questionnaire regarding perceptions on FLHClabor was designed. ii) This questionnaire was applied to to medical students from 18 universities of eight Spanish-speaking Latin American countries. An exploratory factor analysis (EFA) was performed through a principal components analysis with orthogonal varimax rotation. Sample adequacy was evaluated. Factor extraction was based on Kaiser's criteria, Cattell's Scree test and the explained variance $(>5 \%)$. Internal consistency was measured with Cronbach's alpha. Results. 423 students were included in the analysis; $53.4 \%$ were from Peruvian universities. After the EFA, the questionnaire conserved 11 items, which were distributed in three domains, that explaining together $55.47 \%$ of the total variance: i) Perceptions concerning the FLHC physician; ii) Perceptions concerning the FiLC labor and iii) Perceptions about the economic consequences of working in FLHC. Conclusion. The scale is composed by three domains and can be used to assess the perceptions of the medical work on first level of health care of Spanish-speaking Latin American medical students.
\end{abstract}

Key words: Primary health care; Health manpower; Education, medical; Factor analysis, statistical; Latin America (Source: MeSH)

Escuela de Medicina, Universidad Peruana de Ciencias Aplicadas. Lima, Perú

Sociedad Científica de San Fernando, Facultad de Medicina, Universidad Nacional Mayor de San Marcos. Lima, Perú.

Unidad de Análisis y Generación de Evidencia en Salud Pública (UNAGESP), Instituto Nacional de Salud. Lima, Perú.

Sociedad Científica de Estudiantes de Medicina UPC (SOCIEMUPC). Lima, Perú.

Asociación Médica de Investigación y Servicios en Salud. Lima, Perú.

Centro de Excelencia CIGES, Universidad de La Frontera. Temuco, Chile.

Grupo Colaborativo Latinoamericano de Investigación en Recursos Humanos en Salud.

Médico Salubrista, especialista en estadística en investigación, ${ }^{\mathrm{b}}$ médico cirujano, maestro en Ciencias en Epidemiología Clínica, ${ }^{\mathrm{c}}$ estudiante de Medicina,

${ }^{\mathrm{d}}$ médico cirujano, ${ }^{\mathrm{e}}$ matemático, $\mathrm{PhD}$ en Bioestadística

Recibido: 24-04-13 Aprobado: 05-06-13

Citar como: Mayta-Tristán P, Mezones-Holguín E, Pereyra-Elías R, Montenegro-Idrogo JJ, Mejia CR, Dulanto-Pizzorni A, et al. Diseño y validación de una escala para medir la percepción sobre el trabajo en el primer nivel de atención en estudiantes de medicina de Latinoamérica. Rev Peru Med Exp Salud Publica. 2013;30(2):190-6. 


\section{INTRODUCCIÓN}

A más de 30 años de la Declaración de Alma-Ata, existe aún una reconocida necesidad de orientar los sistemas de salud hacia la atención primaria de la salud (APS) $(1,2)$. Esta situación no es ajena a Latinoamérica, donde existen brechas amplias e inequidades fuertemente establecidas, susceptibles de ser solucionadas por la APS ${ }^{(2-4)}$. Para que un sistema de salud orientado a la APS logre resultados, este debería encontrar una base amplia y sólida en el primer nivel de atención (PNA). Este constituye, por lo general, la puerta de entrada al sistema de salud, desde donde se puede organizar la atención integral e integrada que supone la filosofía de la APS ${ }^{(2)}$. Consecuentemente, uno de los pilares para alcanzar los logros perseguidos por la APS supone un incremento, redistribución y disponibilidad adecuada de los recursos humanos en salud (RHUS) que laboren en el primer nivel de atención.

Sin embargo, han sido reconocidos también factores propios de los sistemas de salud, de la formación médica o de motivación personal que pueden conllevar a que el PNA sea percibido como una opción laboral poco atractiva ${ }^{(5-7)}$. Estas impresiones por parte no solo de los médicos sino también de los estudiantes influyen en sus decisiones, pudiendo contribuir en la decisión de no trabajar en el PNA ${ }^{(8)}$.

Para entender el comportamiento en este grupo de recursos humanos jóvenes y en formación, es importante contar con un instrumento que permita conocer y cuantificar las distintas percepciones relacionadas al trabajo en el PNA. Algunos estudios latinoamericanos han intentado valorar la motivación e intención de trabajo en este ámbito ${ }^{(9,10)}$; pero no hemos encontrado instrumentos validados en Latinoamérica que evalúen este aspecto. En dicho contexto, el presente estudio tiene como objetivo diseñar y validar una escala para evaluar la percepción sobre el PNA por parte de los estudiantes de medicina de Latinoamérica.

\section{MATERIALES Y MÉTODOS}

\section{DISEÑO Y POBLACIÓN DE ESTUDIO}

Se realizó un estudio multicéntrico observacional, de corte transversal durante el primer semestre del año 2008, se incluyó a estudiantes de medicina de 18 universidades en ocho países hispanohablantes de Latinoamérica (Chile, Colombia, Ecuador, El Salvador, Honduras, Paraguay, Perú y Venezuela) que se encontraban matriculados en el período académico 2008-1.
Se estimó un tamaño muestral mínimo para validar una escala de 16 ítems en 240 participantes considerando, por lo menos, tener 15 sujetos por ítem. Se usó una muestra no probabilística por conveniencia. Para obtener mayor diversidad de participantes se seleccionó, por lo menos, cuatro alumnos de cada año académico por universidad que no cursen el internado médico (último año de estudios). La información sobre el proceso de selección y características de los sujetos puede verse en una publicación previa ${ }^{(12)}$.

\section{GENERACIÓN DE LA ESCALA}

La elaboración de la escala constó de tres fases, la primera se basó en la identificación de las percepciones de estudiantes, para ello se realizó entrevistas y un grupo focal a estudiantes de medicina de la Universidad Nacional Mayor de San Marcos en Lima, Perú (UNMSM) para identificar las percepciones sobre el trabajo médico en el PNA en cuatro aspectos: características del trabajo, percepciones sobre el médico que trabaja en el PNA; consecuencias económicas y laborales por trabajar en el PNA, y su predisposición para trabajar en el PNA. A partir de este proceso se generaron 24 potenciales ítems por evaluar.

La segunda fase consistió en evaluar el entendimiento de los ítems planteados, de ellos se eliminó ocho por ser muy similares a los que ya estaban planteados, o porque en los pilotos (uno con 40 alumnos de medicina de tercero a quinto año y otro como parte anexa a un estudio con 202 en internos de medicina ${ }^{(12)}$ ) realizados en la UNMSM presentaron alta proporción de no respuesta.

En la tercera fase se realizó la evaluación de la escala de 16 ítems por los colaboradores de 20 universidades de ocho países para que evalúen si los términos usados eran de fácil entendimiento en cada país, luego de ello se procedió a mejorar la redacción de algunos ítems.

Esta escala fue incluida como parte de un cuestionario autoaplicado que evaluó diferentes aspectos sobre las motivaciones de los estudiantes de medicina de Latinoamérica (10). Para cada uno de los 16 ítems se usó una escala de tipo Likert con cinco opciones (totalmente en desacuerdo, en desacuerdo, indiferente, de acuerdo, totalmente de acuerdo) con puntaje del 1 al 5 , considerando el orden mencionado.

Debido a que en las fases previas de la generación de la escala se detectó que los participantes no sabían que era el trabajo médico en el PNA, se incluyó el siguiente encabezado para la escala: Para esta investigación definimos como médicos del PNA a los médicos que laboran en un centro de primer nivel de atención (puestos 
o centros de salud) ya sea en zonas rurales o urbanas, en su país o el extranjero; independientemente de la especialidad (residencia) que piensen o no desarrollar.

\section{ANÁLISIS ESTADÍSTICO}

Se describieron las características de la población en estudio mediante frecuencias absolutas y relativas para las variables categóricas, asi como medidas de tendencia central y dispersión para las variables numéricas.

Se llevó a cabo un análisis factorial exploratorio para establecer la estructura dimensional contenida en los datos obtenidos de la muestra. Para ello, se evaluó inicialmente la matriz de correlación de los ítems y la correlación ítemtest (considerándose como apropiados los valores superiores a 0,3 ). Además, se midió la adecuación muestral global por medio del test de esfericidad de Barlet y se usó el test de Kaiser-Meyer-Olkin (KMO), tomando como un correcto ajuste de la muestra promedio y por ítem cuando los valores fueron superiores a $0,7^{(13)}$.

Se realizó un análisis de componentes principales, debido a que se buscó explicar la varianza total incluyendo la específica, la común y la debida a errores en la medición. Para la selección del número de factores a extraer se contemplaron los criterios de Kaiser (autovalores [eigenvalues] superiores a 1,0), la pendiente de Catell (Gráfico de sedimentación) y la varianza explicada por cada dominio ( $\geq 5 \%$ ). La inclusión de los ítems en sus respectivos dominios fue efectuada en base a los mayores valores de carga estimados (weak loadings), tomando como mínimo un valor de $0,3^{(13)}$. La consistencia interna fue medida con el alpha de Cronbach. El análisis fue efectuado en STATA 12.0 (Stata Corp, Texas, USA).

\section{ASPECTOS ÉTICOS}

Antes de aplicar los cuestionarios, se informó a los participantes acerca del estudio y se les pidió su consentimiento informado verbal. Se les explicó la confidencialidad con la que serían tratados los datos que brindasen. Los cuestionarios fueron anónimos y autoaplicados.

\section{RESULTADOS}

Se encuestó a 435 estudiantes de medicina, se excluyó a 12 participantes que no tenían respuesta en alguno de los ítems de la escala, quedando finalmente 423 observaciones válidas. La mayoría eran varones $(56,0 \%)$ y provenían de Perú $(53,2 \%)$. La distribución por año de estudio fue similar (Tabla 1).
Tabla 1. Características de la población estudiada incluida en el análisis factorial

\begin{tabular}{lc}
\hline Características & $\mathbf{N} .^{\circ}(\%)$ \\
\hline Edad & $21,7(2,7)^{*}$ \\
\hline Sexo & $237(56,0)$ \\
$\quad$ Masculino & $186(44,0)$ \\
\hline Femenino & \\
\hline Años de carrera & $70(16,6)$ \\
\hline Primero & $69(16,3)$ \\
\hline Segundo & $68(16,1)$ \\
\hline Tercero & $81(19,2)$ \\
\hline Cuarto & $75(17,7)$ \\
\hline Quinto & $60(14,2)$ \\
\hline Sexto & \\
\hline País & $225(53,2)$ \\
\hline Perú & $46(10,9)$ \\
\hline Ecuador & $44(10,4)$ \\
\hline Paraguay & $30(7,1)$ \\
\hline Honduras & $25(5,9)$ \\
\hline Chile & $20(4,6)$ \\
\hline Colombia & $19(4,5)$ \\
\hline El Salvador & $14(3,3)$ \\
\hline Venezuela & \\
\hline
\end{tabular}

* Media \pm desviación estándar

Se evaluó los 16 ítems iniciales y se encontró que los ítems $13,14,15$ y 16 , tenían puntaje negativo, por lo que el orden de los puntajes de la escala de Likert fueron invertidas. Tras ello, se observó que todos los ítems presentaron puntaje positivo.

Luego se evidenció que los ítems 8 (Usted considera que la infraestructura es un factor limitante para el desarrollo profesional de un médico de PNA) y 15 (Usted trabajaría en un centro de salud como lugar permanente de trabajo en un país desarrollado) tuvieron una correlación ítemtest inferior a 0,3 , por lo que fueron excluidos del análisis.

Posteriormente, se evaluó que la matriz de correlaciones con los ítems no fue determinante, obteniendo coeficientes de Pearson en su mayoría positivos y solo cuatro negativos. Luego, en el test de esfericidad de Barlett no se detectó inadecuación. Sin embargo, al aplicar el test de KMO global y por ítem, se observó que los ítems 13 (Usted considera que su país requiere más médicos que trabajen en PNA que en hospitales); 14 (Usted trabajaria en un centro de salud como lugar permanente de trabajo en su país), y 16 (Usted cree que en 10 años podría estar trabajando en un centro de salud) no alcanzaban el puntaje KMO mínimo $(0,7)$ por lo que fueron excluidos del 
Tabla 2. Análisis de adecuación de la muestra para realizar el análisis factorial con el método de Kaiser-Meyer-Olkin

\begin{tabular}{|c|c|c|c|c|c|c|}
\hline Ítem $* *$ & $\begin{array}{l}\text { Valor de } \\
\text { carga }\end{array}$ & Factor & $\begin{array}{l}\text { Correlación } \\
\text { ítem-test }\end{array}$ & Alpha & Media $†$ & KMO \\
\hline $\begin{array}{l}\text { 1. Usted cree que un médico que trabaja en el PNA tiene menos } \\
\text { prestigio ante la sociedad que un médico que trabaja en un hospital. }\end{array}$ & 0,68 & 1 & 0,62 & 0,76 & 2,51 & 0,88 \\
\hline $\begin{array}{l}\text { 2. Usted considera que el médico que trabaja en el PNA es porque no } \\
\text { tuvo otra opción laboral. }\end{array}$ & 0,77 & 1 & 0,60 & 0,76 & 2,50 & 0,83 \\
\hline $\begin{array}{l}\text { 3. Usted considera que el médico que trabaja en el PNA tiene menor } \\
\text { preparación académica que el que trabaja en un hospital. }\end{array}$ & 0,78 & 1 & 0,61 & 0,76 & 2,34 & 0,83 \\
\hline $\begin{array}{l}\text { 4. Usted considera que un médico que labora en el PNA tiene un menor } \\
\text { ingreso económico que un médico que labora en un hospital. }\end{array}$ & 0,74 & 3 & 0,47 & 0,77 & 3,59 & 0,77 \\
\hline $\begin{array}{l}\text { 5. Usted considera que el médico que trabaja en el PNA tiene un menor } \\
\text { estatus dentro del gremio médico, en comparación con los que } \\
\text { trabajan en un hospital. }\end{array}$ & 0,57 & 1 & 0,71 & 0,74 & 2,96 & 0,87 \\
\hline $\begin{array}{l}\text { 6. Usted considera que si trabajase en el PNA en su país, no podría } \\
\text { satisfacer sus necesidades económicas. }\end{array}$ & 0,80 & 3 & 0,53 & 0,77 & 3,39 & 0,79 \\
\hline $\begin{array}{l}\text { 7. Para usted el trabajo en el PNA es un período de transición entre el } \\
\text { terminar la carrera y la especialización (residencia). }\end{array}$ & 0,33 & 1 & 0,49 & 0,77 & 3,32 & 0,86 \\
\hline $\begin{array}{l}\text { 9. Usted considera que en el PNA se ven casos médicos poco } \\
\text { interesantes, en relación con la actividad hospitalaria. }\end{array}$ & 0,76 & 2 & 0,51 & 0,77 & 3,01 & 0,78 \\
\hline $\begin{array}{l}\text { 10. Usted considera que el trabajo en el PNA es rutinario, en relación } \\
\text { con la actividad hospitalaria. }\end{array}$ & 0,82 & 2 & 0,55 & 0,76 & 3,16 & 0,72 \\
\hline $\begin{array}{l}\text { 11. Usted considera que el trabajo en el PNA es muy limitado, en } \\
\text { relación con la actividad hospitalaria. }\end{array}$ & 0,76 & 2 & 0,59 & 0,76 & 3,49 & 0,79 \\
\hline $\begin{array}{l}\text { 12. Usted considera que la formación académica que ha recibido en la } \\
\text { universidad está orientada más a un trabajo hospitalario que del PNA. }\end{array}$ & 0,31 & 2 & 0,46 & 0,78 & 3,48 & 0,87 \\
\hline Global & & & & 0,78 & & 0,81 \\
\hline
\end{tabular}

* Todos los ítems tuvieron signo positivo

** Los ítems 8 y 15 fueron excluidos por tener una correlación ítem-test menor de 0,3 y los ítems 13, 14 y 16 por tener un KMO menor de 0,7

$\dagger$ Media de los puntajes obtenidos

análisis. Con los 11 ítems restantes, se obtuvo un puntaje promedio global de 0,81 y todos los puntajes de cada ítem fueron superiores a 0,7; oscilando entre 0,72 a 0,88 (Tabla 2). Los textos mencionados previamente para los ítems $8,13,14,15$ y 16, así como los que aparecen en la Tabla 2 son los fraseos original de los ítems incluidos en la escala original.

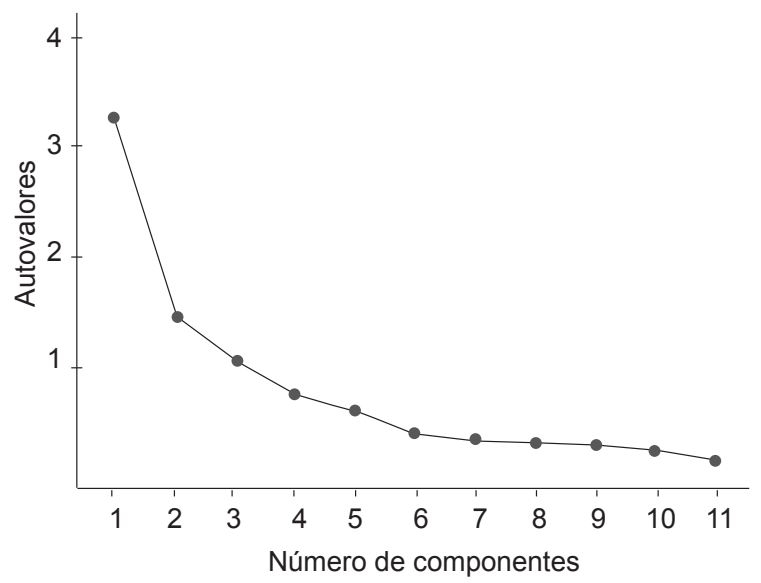

Figura 1. Gráfico de sedimentación posterior al análisis de componentes principales con los 11 ítems incluidos
Tras ello, se realizó la extracción de factores por medio de un análisis de componentes principales. Basado en el criterio de Kaiser, gráfico de sedimentación (Figura 1) y la varianza explicada por cada dominio, se identificó tres dominios que explicaban el 55,5\% de la varianza (Tabla 3). Posteriormente, tras la rotación ortogonal varimax se identificó los ítems que correspondían a cada uno de los dominios identificados.

En la Tabla 2 se observa la distribución de ítems por cada uno de los tres dominios, así como la consistencia interna global de la escala con los 11 ítems incluidos y el puntaje específico para cada ítem si este fuera retirado; se observa que en todos los casos es mayor a 0,7. Asimismo, la correlación entre los dominios extraídos fue superior a 0,3 en todos los casos. La consistencia interna global fue alta (alfa=0,78).

\section{DISCUSIÓN}

La escala generada muestra una potencial utilidad para evaluar las percepciones en distintas esferas respecto al trabajo en el PNA en estudiantes de medicina 
Tabla 3. Autovalores (eigenvalues) y proporción de varianza explicada para los tres dominios de extracción inicial tras el análisis de componentes principales, y posterior a la rotación varimax (11 ítems)

\begin{tabular}{|c|c|c|c|c|c|c|c|}
\hline \multirow{2}{*}{$\begin{array}{c}\text { Dominios } \\
\text { (percepciones sobre ...) }\end{array}$} & \multicolumn{3}{|c|}{$\begin{array}{l}\text { Análisis de componentes } \\
\text { principales }\end{array}$} & \multicolumn{3}{|c|}{ Rotación Varimax } & \multirow{2}{*}{ Alfa } \\
\hline & $\begin{array}{c}\text { Autovalor } \\
\text { total }\end{array}$ & Diferencia & $(\%)^{*}$ & $\begin{array}{l}\text { Autovalor } \\
\text { total }\end{array}$ & Diferencia & $(\%)^{*}$ & \\
\hline $\begin{array}{l}\text { Factor 1: el médico que trabaja en el } \\
\text { PNA. }\end{array}$ & 3,50 & 1,97 & $(31,8)$ & 2,20 & 0,12 & $(20,0)$ & 0,73 \\
\hline $\begin{array}{l}\text { Factor 2: el trabajo asistencial en el } \\
\text { PNA. }\end{array}$ & 1,53 & 0,46 & $(13,9)$ & 2,08 & 0,26 & $(18,9)$ & 0,65 \\
\hline $\begin{array}{l}\text { Factor 3: consecuencias económicas de } \\
\text { trabajar en el PNA. }\end{array}$ & 1,07 & -- & $(9,8)$ & 1,82 & --- & $(16,5)$ & 0,60 \\
\hline
\end{tabular}

* Porcentaje de varianza explicada, en ambos casos la global fue de $55,5 \%$

latinoamericanos hispanohablantes. Esta escala podría servir para realizar evaluaciones locales más amplias sobre esta realidad. Subsecuentemente, el conocer estas apreciaciones permitiría realizar las intervenciones o reformas específicas necesarias para lograr revertir una situación desfavorable para la fuerza laboral en el PNA, posibilidad que ya ha sido contemplada previamente en otras latitudes ${ }^{(8,14-17)}$.

Es importante recalcar que este instrumento contiene ítems que reflejan solamente percepciones, las cuales pueden ser realidades o creencias, y pueden variar según cada contexto, de acuerdo con la posición que ocupa el médico que labora en el PNA propiamente dicha en el sistema de salud de cada país. En ese sentido, algunos de los ítems nos pueden mostrar la magnitud con la que están arraigados algunos mitos para así poder mitigarlos con educación y motivación en las facultades y escuelas de medicina con el fin de incrementar el interés de los estudiantes por el PNA ${ }^{(8,14,15)}$. Asimismo, los ítems que revelen una realidad deficiente del sistema de salud pueden ser sujetos a cambio a través de una mejora de las políticas públicas locales ${ }^{(18-20)}$.

Esta escala tiene una puntuación global en forma numérica, con calificaciones que van desde los 11 hasta los 55 puntos, donde el puntaje 11 equivale a una percepción positiva del trabajo médico en el PNA y el puntaje 55 a una percepción muy negativa. Esta escala presenta tres dominios: i) Percepciones sobre el médico que trabaja en el PNA (puntaje del 5 al 25); ii) Percepciones sobre el trabajo asistencial en el PNA (puntaje del 4 al 20), y iii) Percepciones sobre las consecuencias económicas de trabajar en el PNA (puntaje del 2 al 10). Asimismo, las puntuaciones en los dominios denotan intensidad solamente. No es objetivo de la escala global ni de los dominios tener una función discriminante.

El primer dominio de la escala agrupa a las percepciones sobre el médico que labora en el PNA; las cuales pueden variar entre escenarios. Investigaciones previas en estudiantes canadienses y suizos revelan que el médico del PNA tiene un menor prestigio ante la sociedad y el gremio médico respecto a los otros especialistas ${ }^{(16,21,22)}$. También se ha encontrado que es visto como "poco preparado" o con "pobres competencias" (21-23), o que se encuentra en una transición entre el término del pregrado y el inicio de una especialización (diferente de medicina familiar) ${ }^{(5)}$. Entretanto, una muestra de más de cinco mil estudiantes pertenecientes a casi todas las universidades de España percibe al médico del PNA con mucha mayor consideración en distintas esferas ${ }^{(17)}$.

En el segundo dominio, convergen todos los ítems relacionados a las percepciones sobre el trabajo en el PNA propiamente dicho. Estudios en Suiza y Hungría han encontrado que estas incluyen ver casos clínicos poco interesantes o serios ${ }^{(21,24)}$. En contraste, los estudiantes finlandeses conciben que el trabajo en el PNA es versátil y lleno de desafíos ${ }^{(13)}$. En ese mismo sentido, se aprecian otras limitaciones como las reducidas posibilidades de tratamiento y manejo, las deficiencias en la infraestructura o la pobre inversión recibida en general para el trabajo en el PNA ${ }^{(15,24,25)}$. Del mismo modo, puede que no se perciban limitaciones en otras áreas del trabajo del PNA, como la relación médico-paciente, la cercanía, integralidad y continuidad y longitudinalidad en la atención ${ }^{(8,16,26,27)}$.

Dentro del segundo dominio, también se encuentra la percepción acerca de la formación académica recibida en sus respectivas escuelas de medicina. En distintos contextos, e incluso en países desarrollados con un sistema basado en el PNA como Canadá, se han reportado fuertes percepciones de motivación formativa orientada hacia una especialización de trabajo hospitalario ${ }^{(8,21)}$. Como contraparte, experiencias de entrenamiento en el PNA en el pregrado influyen positivamente en la elección de esta especialidad ${ }^{(28,29)}$. En Latinoamérica, la formación médica de pregrado es orientada principalmente al trabajo 
hospitalario, con pocas horas de formación y exposición al trabajo en el PNA ${ }^{(30)}$.

El tercer dominio se refiere a las percepciones sobre la economía relativa al trabajo en el PNA. Los salarios obtenidos por los médicos del PNA son percibidos como menores respecto de otras áreas de la medicina asistencial ${ }^{22}$, en países donde los alumnos se endeudan para estudiar la carrera es conocido que el trabajo en el PNA es preferido por satisfacer más rápidamente sus necesidades económicas comparado con otras especialidades, ya que requieren postular y trabajar primero como residentes para pagar deudas de estudios ${ }^{(31)}$.

A pesar de la diversidad existente en los distintos países de Latinoamérica estudiados, al ser sometida al análisis, la escala ha demostrado funcionar adecuadamente. Este instrumento puede además orientar acerca de la variación de las percepciones sobre el trabajo en el PNA a lo largo de los años de estudio, luego de la exposición a asignaturas que incluyan componentes del PNA o al sistema de salud vigente local en donde se realizan las rotaciones clínicas formativas en la carrera de medicina.

El estudio presenta ciertas limitaciones, como la mayor proporción de estudiantes peruanos en la muestra analizada, lo cual podría implicar un sesgo de selección generando una mayor influencia de estos sujetos de estudio en el comportamiento de la escala. Sin embargo, realizamos paralelamente (resultados no presentados) el análisis solo con los participantes que no eran de Perú y se encontraron los mismos resultados que los aquí mostrados.

Se concluye que la escala generada a través de este estudio puede ser usada para medir las percepciones de los estudiantes de medicina latinoamericanos de habla hispana respecto del primer nivel de atención y está compuesta por tres dominios: i) Percepciones sobre el médico que trabaja en el PNA; ii) Percepciones sobre el trabajo asistencial en el PNA, y iii) Percepciones sobre las consecuencias económicas de trabajar en el PNA. Este instrumento se presenta como una posibilidad para conocer más claramente cómo ve esta población al PNA, para así intervenir selectivamente sobre las variables apropiadas en busca de "convencerlos" (32) de trabajar en el PNA, tan necesaria para mejorar las condiciones sanitarias de nuestras poblaciones ${ }^{(2)}$.

Fuente de financiamiento: autofinanciado

Conflictos de interés: los autores declaran no tener conflictos de interés.

Contribución de autoría: PMT tuvo la idea de investigación y realizó la supervisión de la investigación, PMT y ADP elaboraron el instrumento, EMH y SRM realizaron el análisis de datos, PMT, RPE, CRM, JJMI y EMH interpretaron los resultados, RPE, CRM, EMH y JJMI escribieron el borrador del manuscrito, PMT, ADP, CRM, SRM y EMH revisaron críticamente el manuscrito, todos los autores aprobaron la versión final a publicar.

Agradecimientos: a cada uno de los participantes de Red-LIRHUS que contribuyeron al desarrollo de esta investigación: Christian Adrián López-Castillo (Colombia); Daniela Sanhueza y Natalia Peña (Chile); Fabio Nogueira (Brasil); Jorge A. Barrezueta (Ecuador); Beatriz Deras (El Salvador); Lysien I. Zambrano (Honduras); Gustavo Codas (Paraguay); Jhonatan Losanto (Paraguay); Manuel Alejandro Rodríguez (Venezuela); Danny CarbajalGonzález, Javier Villafuerte-Gálvez, Caleb Llactamaray, C. Hugo Arroyo-Hernández, Lorena Escalante-Romero, Renato Farfán, Julio Maquera-Afaray, y George Hernández (Perú).

\section{REFERENCIAS BIBLIOGRÁFICAS}

1. World Health Organization. Working together for health: the world health report 2006. Geneva: WHO; 2006.

2. World Health Organization. The world health report: primary health care now more than ever. Geneva: WHO; 2008.

3. Roses Periago M. Renewing primary health care in the Americas: the Pan American Health Organization proposal for the twenty-first century. Rev Panam Salud Publica. 2007;21(23):65-72.

4. Ventres WB. The emergence of primary care in Latin America: reflections from the field. J Am Board Fam Med. 2013;26(2):183-6.

5. Buddeberg-Fischer B, Klaghofer R, Stamm M, Marty F, Dreiding $\mathrm{P}$, Zoller $\mathrm{M}$, et al. Primary care in Switzerland--no longer attractive for young physicians? Swiss Med Wkly. 2006; 136:416-24.

6. Wright S, Wong A, Newill C. The impact of role models on medical students. J Gen Intern Med. 1997;12(1):53-6.

7. Serneels P, Montalvo JG, Pettersson G, Lievens T, Butera JD, Kidanu
A. Who wants to work in a rural health post? The role of intrinsic motivation, rural background and faith-based institutions in Ethiopia and Rwanda. Bull World Health Organ. 2010;88(5):342-9.

8. Rodríguez C, Tellier PP, Bélanger E. Exploring professional identification and reputation of family medicine among medical students: a Canadian case study. Educ Prim Care. 2012;23(3):158-68.

9. Ramírez M. Expectativas profesionales de internos de medicina y su inclinación 
por la atención primaria de salud. An Fac Med (Lima). 2008;69(3):176-81.

10. Carrera LI, Enría GT, D’Ottavio AE. La atención primaria de la salud y especialización médica: ¿Categoría opuestas o complementarias? Educ Med. 2004:7(4):132-39.

11. Mayta-Tristán P, Carbajal-Gonzalez D, Mezones-Holguín E, Mejia CR, Pereyra-Elías R, VillafuerteGálvez, et al. Situación actual y perspectivas profesionales de los estudiantes de medicina de nueve países de Latinoamérica, 2008: estudio preliminar. CIMEL. 2010;15(1):3-8.

12. Mayta-Tristán P, Dulanto-Pizzorni A. Prevalencia y factores asociados con la intención de emigración en internos de medicina de una universidad pública, Lima 2007. Rev Peru Med Exp Salud Publica. 2008;25(3):274-8.

13. Pett M, Lackey N, Sullivan J. Making sense of factor analysis: the use of factor analysis for instrument development in health care research. California: Sage Publication; 2003.

14. Kuikka L, Nevalainen MK, Sjöberg L, Salokekkilä P, Karppinen H, Torppa $\mathrm{M}$, et al. The perceptions of a GP's work among fifth-year medical students in Helsinki, Finland. Scand J Prim Health Care. 2012;30(2):121-6.

15. Phillips J, Weismantel D, Gold K, Schwenk T. How do medical students view the work life of primary care and specialty physicians? Fam Med. 2012;44(1):7-13.

16. Scott I, Gowans M, Wright B, Brenneis F, Banner S, Boone J. Determinants of choosing a career in family medicine. CMAJ. 2011;183(1):E1-8.

17. Zurro AM, Villa JJ, Hijar AM, Tuduri XM, Puime ÁO, Alonso-Coello P, et al. Medical student attitudes towards family medicine in Spain: a statewide analysis. BMC Fam Pract. 2012;13:47.
18. Casado Vicente V, Bonal Pitz P, Cucalón Arenal JM, Serrano Ferrández E, Suárez Gonzalez F. La medicina familiar y comunitaria y la universidad. Informe SESPAS 2012. Gac Sanit. 2012;26 Suppl 1:69-75.

19. González López-Valcárcel B, Barber Pérez P. Planificación y formación de profesionales sanitarios, con foco en la atención primaria. Informe SESPAS 2012. Gac Sanit. 2012;26 Suppl 1:46-51.

20. Bärnighausen T, Bloom DE. Financial incentives for return of service in underserved areas: a systematic review. BMC Health Services Research. 2009;9(86):1-17.

21. Buddeberg-Fischer B, Stamm M, Marty F. Family medicine in Switzerland: training experiences in medical school and residency. Fam Med. 2007;39(9):651-5.

22. Gill H, McLeod S, Duerksen K, Szafran O. Factors influencing medical students' choice of family medicine: effects of rural versus urban background. Can Fam Physician. 2012;58(11):e649-57.

23. Buddeberg-Fischer B, Stamm M, Buddeberg C, Klaghofer R. The new generation of family physicians-career motivation, life goals and work-life balance. Swiss Med Wkly. 2008;138:305-12.

24. Girasek E, Eke E, Szócska M. Analysis of a survey on young doctors' willingness to work in rural Hungary. Hum Resour Health. 2010;8:13

25. van der Voort CT, van Kasteren G, Chege P, Dinant GJ. What challenges hamper Kenyan family physicians in pursuing their family medicine mandate? A qualitative study among family physicians and their colleagues. BMC Fam Pract. 2012;13:32.

26. Kiolbassa K, Miksch A, Hermann $\mathrm{K}$, Loh A, Szecsenyi J, Joos S, et al. Becoming a general practitioner-- which factors have most impact on career choice of medical students? BMC Fam Pract. 2011 May 9; 12:25.

27. Buddeberg-Fischer B, Klaghofer R, Stamm M. Family physicians in Switzerland: transition from residency to family practice. Fam Med. 2011;43(1):29-36.

28. Dick JF 3rd, Wilper AP, Smith S, Wipf $\mathrm{J}$. The effect of rural training experiences during residency on the selection of primary care careers: a retrospective cohort study from a single large internal medicine residency program. Teach Learn Med. 2011;23(1):53-7.

29. Tate RB, Aoki FY. Rural practice and the personal and educational characteristics of medical students: survey of 1269 graduates of the University of Manitoba. Can Fam Physician. 2012;58(11):e641-8.

30. Knox L, Ceitlin J, Hahn RG. Slow progress: predoctoral education in family medicine in four Latin American countries. Fam Med. 2003;35(8):591-5.

31. Vanasse A, Orzanco MG, Courteau J, Scott S. Attractiveness of family medicine for medical students: influence of research and debt. Can Fam Physician. 2011;57(6):e216-27.

32. Deutsch T, Hönigschmid P, Wippermann U, Frese T, Sandholzer H. Don't select medical students--convince them. CMAJ. 2011;183(17):2017.

Correspondencia: Percy Mayta-Tristán Dirección: Av. Brasil 2169 Dpto. 802, Lima 11, Perú.

Teléfono: (511) 987532133

Correoelectrónico:percy.mayta@upc.edu.pe 